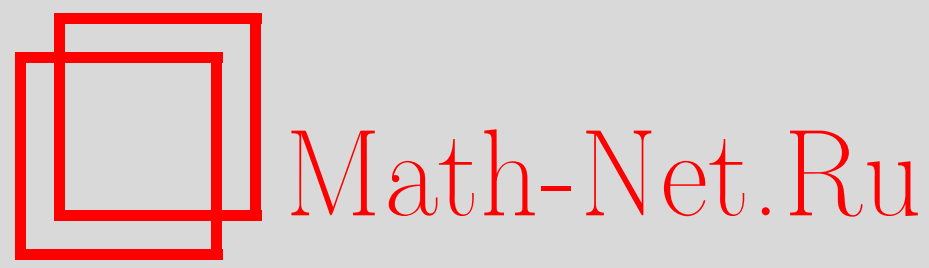

М. В. Карасёв, Представление оператора эволюции через мембранные амплитуды, Матем. заметки, 1996, том 60, выпуск 6, 930-934

DOI: https://doi.org/10.4213/mzm1912

Использование Общероссийского математического портала MathNet.Ru подразумевает, что вы прочитали и согласны с пользовательским соглашением

http://www . mathnet.ru/rus/agreement

Параметры загрузки:

IP : 54.172 .240 .79

26 апреля 2023 г., 12:28:28

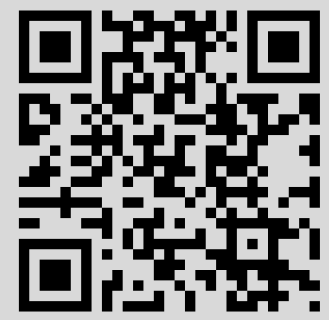




\section{ПРЕДСТАВЛЕНИЕ ОПЕРАТОРА ЭВОЛЮЦИИ ЧЕРЕЗ МЕМБРАННЫЕ АМПЛИТУДЫ}

\section{B. Карасев}

1. Мембранные амплитуды в виковском квантовании. Мы используем здесь схему квантования, основанную на понятии когерентного состояния [1], [2] . Пусть $\mathscr{L}$ - некоторое гильбертово пространство, а $\mathfrak{X}=\mathfrak{X}^{2 n}$ - связное и односвязное многообразие с комплексной структурой (т.е. с локальными комплексньми координатами $\left.z=\left(z^{1}, \ldots, z^{n}\right)\right)$. Пусть $\Pi: \mathfrak{X} \rightarrow \operatorname{Hom}(\mathscr{L})$ - гладкое невырожденное отображение, удовлетворяющее условиям:

I) для любого $x \in \mathfrak{X}$ оператор $\Pi(x)$ является ортогональным проектором на пространство, натянутое на некоторый ненулевой вектор $\pi(z(x)) \in \mathscr{L}$, причем семейство $\pi(z)$ локально голоморфно;

II) имеет место разложение единицы: $\int_{\mathfrak{X}} \Pi(x) d m(x)=\mathbf{I}$ (слабый интеграл) для некоторой меры $d m$ на $\mathfrak{X}$;

III) линейная оболочка проекторов $\{\boldsymbol{\Pi}(x) \mid x \in \mathfrak{X}\}$ плотна в алгебре операторов Гильберта-Шмидта на $\mathscr{L}$.

Будем говорить, что П - это квантование комплексного многообразия $\mathfrak{X}$ над гильбертовым пространством $\mathscr{L}$. На естественных классах функций $f$ над $\mathfrak{X}$ можно определить отображение $f \rightarrow \widehat{f} \stackrel{\text { def }}{=} \int_{\mathfrak{X}} f(x) \Pi(x) d m(x)$. Таким образом, $\widehat{f}$ - это некоторый линейный оператор в $\mathscr{L}$. Функция $f$ называется антивиковским (или контравариантным, или верхним) символом оператора $\widehat{f} ;$ функция $x \rightarrow \operatorname{tr}(\widehat{f} \Pi(x))$ называется виковским символом оператора $\widehat{f}$ (или ковариантным, или нижним); векторы $\pi(z)$ назьваются (голоморфными) когерентнылии состояниями [1]-[5]. Отметим, что аксиома III, введенная в [6], не встречалась в традиционных вариантах.

В [6]-[10] была развита конструкция квантования, основанная на понятии комплексного группоида, мембранных амплитуд и геометрических когерентных состояний над лагранжевыми подмногообразиями; в данной работе приведены некоторые следствия из этой общей конструкции.

Итак, пусть задано некоторое квантование П многообразия $\mathfrak{X}$. Введем на $\mathfrak{X}$ кэлерову метрику и соответствующую 2-форму

$$
g_{\bar{\alpha} \beta}=\frac{\partial^{2}}{\partial \bar{z}^{\alpha} \partial z^{\beta}} \ln \|\pi(z)\|_{\mathscr{L}}^{2}, \quad \omega=i \sum_{\alpha, \beta=1}^{n} g_{\bar{\alpha} \beta} d \bar{z}^{\alpha} \wedge d z^{\beta} .
$$

Форма $\omega$ аналитически продолжается на комплексный группоид, соответствующий многообразию $\mathfrak{X}$, т.е. на прямое произведение $\mathfrak{X} \times \mathfrak{X}$ (которое может рассматриваться как один из возможных вариантов комплексификации многообразия $\mathfrak{X}$; см. детали в [6], [8], [9]). Продолжение задается явной формулой:

$$
\omega_{\left(x, x^{\prime}\right)}=i \sum_{\alpha, \beta=1}^{n} \frac{\partial}{\partial \bar{z}^{\prime \alpha}} \frac{\partial}{\partial z^{\beta}} \ln \left(\pi(z), \pi\left(z^{\prime}\right)\right)_{\mathscr{L}} d \bar{z}^{\prime \alpha} \wedge d z^{\beta},
$$


где $z=z(x), z^{\prime}=z\left(x^{\prime}\right),\left(x, x^{\prime}\right) \in \mathfrak{X} \times \mathfrak{X}$. На диагонали $\operatorname{diag}(\mathfrak{X} \times \mathfrak{X}) \approx \mathfrak{X}$, т.е. при $x=x^{\prime}$, форма (1.2) совпадает с (1.1), а на подмножестве точек, сопряженных относительно метрики $g$, эта форма имеет особенности.

Ниже, в п. 3 мы будем дополнительно предполагать, что метрика $g$ представима в виде

$$
g=g_{0}+\bar{\partial} \partial \ln \operatorname{det} g_{1} / 2,
$$

где $g_{0}$ и $g_{1}$ - некоторые кэлеровы метрики на $\mathfrak{X}$. Основной вывод из результатов [6]-[10], состоит в том, что через эти метрики и через соответствующие им 2-формы могут быть представлены квантовые (некоммутативные) деформации алгебр функций над фазовым многообразием $\mathfrak{X}$, а также точные и квазиклассические решения квантовых уравнений (типа Шрёдингера, волнового, диффузии и др.) в гильбертовом пространстве $\mathscr{L}$.

Ключевое наблюдение состоит в следующем. Для любых $x, y \in \mathfrak{X}$ обозначим через $\Sigma(x, y)$ двумерную пленку (мембрану) в группоиде $\mathfrak{X} \times \mathfrak{X}$, граница которой составлена из отрезков путей

$$
(x, x) \rightarrow(x, y) \rightarrow(y, y) \rightarrow(y, x) \rightarrow(x, x) .
$$

Здесь каждая из стрелок $(\rightarrow)$ обозначает произвольный путь вдоль "горизонтали" или “вертикали" в прямом произведении $\mathfrak{X} \times \mathfrak{X}$. Имеет место следующая формула:

$$
p(x, y) \stackrel{\text { def }}{=} \operatorname{tr}(\Pi(x) \Pi(y))=\exp \left\{-2 \Im \int_{\Sigma(x, y)} \omega\right\}
$$

где $\Im$ обозначает взятие мнимой части. В правой части здесь появилась одна из простейших мембранных амплитуд с четырехугольной границей (1.4) (в других ситуациях амплитуды строятся по мембранам с более сложной конфиигурацией [7]-[10]). Она называется вероятностной функцией. Это гладкая симметричная функция на $\mathfrak{X} \times \mathfrak{X}$, принимающая значения между 0 и 1 , причем $\int_{\mathfrak{X}} p(x, y) d m(x)=1$ для любого $y$. Интегральный оператор $\mathscr{P}$ с ядром $p(x, y)$ переводит антивиковские символы в виковские. Ортогональное дополнение в $L^{2}(\mathfrak{X}, d m)$ к нуль-подпространству оператора $\mathscr{P}$ задает квантовую алгебру $\mathscr{F}$ функций на $\mathfrak{X}$ (антивиковских символов), изоморфную алгебре операторов Гильберта-Шмидта в $\mathscr{L}$. Умножение в $\mathscr{F}$ описывается геометрически с помошью функи,ии голономии, построенной по мембране с шестиугольной границей (см. [6], [8], [9]).

2. Геометрическое представление интеграла Клаудера. Используя мембранные амплитуды, можно чисто геометрически выразить виковский символ композиции нескольких операторов через антивиковские символы сомножителей и получить отсюда новое представление для интегралов по путям типа Клаудера [11], [12]. Пункт (а) следующей теоремы взят из [8], [9].

ТЕОРема 2.1. (а) Виковский символ композичии вычисляется по формулe

$$
\begin{aligned}
\operatorname{tr} & \left(\widehat{f}_{1} \cdots \widehat{f}_{N} \Pi(x)\right) \\
& =\int_{\mathfrak{X}} \cdots \int_{\mathfrak{X}} \exp \left\{i \int_{\Sigma\left(x_{N}, \ldots, x_{1}, x\right)} \omega\right\} f\left(x_{N}\right) \cdots f\left(x_{1}\right) d m\left(x_{N}\right) \cdots d m\left(x_{1}\right) .
\end{aligned}
$$


Через $\Sigma\left(x_{N}, \ldots, x_{1}, x\right)$ обозначена мембрана в $\mathfrak{X} \times \mathfrak{X}$ со следующей граничей:

$$
\begin{aligned}
(x, x) \rightarrow\left(x, x_{1}\right) \rightarrow\left(x_{1}, x_{1}\right) & \rightarrow\left(x_{1}, x_{2}\right) \rightarrow\left(x_{2}, x_{2}\right) \\
& \rightarrow \cdots \rightarrow\left(x_{N}, x_{N}\right) \rightarrow\left(x_{N}, x\right) \rightarrow(x, x),
\end{aligned}
$$

где каждая из стрелок ' $\rightarrow$ ' обозначает отрезок пути вдоль "горизонтали" или "вертикали" в әруппоиде $\mathfrak{X} \times \mathfrak{X}$.

(b) Пусть $X=\{X(\tau) \mid 0 \leqslant \tau \leqslant 1\}-$ гёльдеров путь на многообразии $\mathfrak{X}$, и пусть $x_{j}=X(j / N)(j=0, \ldots, N)$ - точки разбиения этого пути. Тогда nрu $N \rightarrow \infty$

$$
\exp \left\{i \int_{\Sigma\left(x_{N}, \ldots, x_{1}, x_{0}\right)} \omega\right\} \simeq \exp \left\{i \int_{\Sigma[X]} \omega\right\} \cdot \exp \left\{-\frac{1}{4} \sum_{j=1}^{N-1} d\left(x_{j+1}, x_{j}\right)^{2}\right\} \text {. }
$$

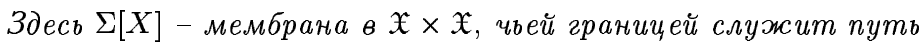

$$
(X(0), X(0)) \stackrel{X}{\rightrightarrows}(X(1), X(1)) \rightarrow(X(1), X(0)) \rightarrow(X(0), X(0))
$$

(причем звено, обозначенное двумя стрелками '马', идет вдоль пути $X$ по диагонали $\operatorname{diag}(\mathfrak{X} \times \mathfrak{X}) \approx \mathfrak{X})$. Кроме того, в (2.2) участвует риманово расстояние $d$, заданное метрикой $g(1.1)$.

Определим теперь элемент объема $d \mathfrak{M}$ в пространстве всевозможных путей $X:[0,1] \rightarrow \mathfrak{X}$ следующим образом:

$$
d \mathfrak{M}[X] \stackrel{\text { def }}{=} \lim _{N \rightarrow \infty} \Re\left(\exp \left\{i \int_{\Sigma\left(x_{N}, \ldots, x_{1}, x_{0}\right)} \omega\right\}\right), \quad x_{j}=X(j / N),
$$

где $\Re$ обозначает взятие вещественной части. В силу теоремы 2.1 (b) значения этого элемента объема на пространстве гёльдеровых путей можно вычислять по формуле

$$
\begin{aligned}
d \mathfrak{M}[X]= & p(X(0), X(1))^{1 / 2} \cos \left(\Re \int_{\Sigma[X]} \omega\right) \\
& \times \prod_{\tau=0}^{t} d m(X(\tau)) e^{-d(X(\tau+d \tau), X(\tau))^{2} / 4},
\end{aligned}
$$

где $p$ - вероятностная функция (1.5), мембрана $\Sigma[X]$ определена в теореме $2.1(\mathrm{~b})$, форма $\omega$ - в (1.1), а $d$ - риманово расстояние на $\mathfrak{X}$, заданное метрикой (1.1).

Отметим, что лишा на гладких путях $X$ гауссовы экспоненты в правых частях (2.2), (2.4) можно заменить единицей. Такие экспоненты не встречались ни в традиционных подходах к фейнмановским интегралам, ни в тех, которые основаны на теории когерентных состояний (см., например, [11]-[13]). Они сушественно улучшают свойства элемента объема $d \mathfrak{M}$ и приближают их к свойствам настоящей меры в пространстве путей (подобной мере Винера).

Обратим также внимание на дополнительные множители в $(2.4)$, связанные с интегралом по мембране $\Sigma[X]$ и с вероятностной функцией $p(X(0), X(1))$. Эта последняя аппроксимирует $\delta$-функцию с носителем в точке $X(0)=X(1)$ так, что элемент объема $d \mathfrak{M}$ оказывается квазиклассически сосредоточенным на замкнутых путях $X$.

Теперь мы применим формулы (2.1), (2.2) для вычисления виковского символа оператора эволюции и виковского символа проекторов на собственные подпространства некоторого гамильтониана $\widehat{H}$ с гладким вещественным антивиковским символом $H$. Пусть для простоты $\mathfrak{X}$ компактно. 
ТЕОрема 2.2. (а) Виковский символ оператора $e^{-i t \widehat{H}}$ задается с помощью интеграла по путям (понимаемого как предел конечнократных аппроксимачий):

$$
\operatorname{tr}\left(e^{-i t \widehat{H}} \boldsymbol{\Pi}(x)\right)=\int_{X(0)=x} e^{-i t H[X]} d \mathfrak{M}[X]
$$

Здесь интегрирование ведется по путям $X=\{X(\tau) \mid 0 \leqslant \tau \leqslant 1\}$ на многообразии $\mathfrak{X}$, начинающимся в точке $x=X(0)$. Использовано такжсе следующее обозначение: $H[X]=\int_{0}^{1} H(X(\tau)) d \tau$. В частности, имеем: $\int_{X(0)=x} d \mathfrak{M}[X]=1$.

(b) Число $\lambda$ является собственным значением оператора $\widehat{H}$ тогда и только тогда, когда интеграл $\int_{H[X]=\lambda} d \mathfrak{M}[X] \stackrel{\text { def }}{=} n^{\lambda}$ отличен от нуля (здесь интегрирование ведется по всем путям $X$, для которых $H[X]=\lambda$ ). В этом случае $n^{\lambda}$ совпадает с кратностью собственного значения $\lambda$, т.е. $n^{\lambda}=\operatorname{dim} P^{\lambda}$. Виковский символ соответствующего собственного проектора $P^{\lambda}$ (или функиия Хусими) принимает положстельнье значения межсду 0 и 1 и задается формулой

$$
\operatorname{tr}\left(P^{\lambda} \Pi(x)\right)=\int_{X(0)=x, H[X]=\lambda} d \mathfrak{M}[X] .
$$

Интегрируя левые и правые части по $x \in \mathfrak{X}$ с некоторой функцией $f$, получаем

СлеДСтвиЕ 2.1. (а) Для любого $f \in C_{0}^{\infty}(\mathfrak{X})$ имеет место формула следа

$$
\operatorname{tr}\left(e^{-i t \widehat{H}} \widehat{f}\right)=\int e^{-i t H[X]} f(X(0)) d \mathfrak{M}[X],
$$

где интегрирование ведется по всевозмохсным путям $X$ в $\mathfrak{X}$. В частнос$m u, \int d \mathfrak{M}=\operatorname{dim} \mathscr{L}=\int_{\mathfrak{X}} d m$.

(b) Mатричные әлементы оператора $\widehat{f}$ на собственных подпространствах оператора $\widehat{H}$ задаются формулой

$$
\operatorname{tr}\left(\widehat{f} P^{\lambda}\right)=\int_{H[X]=\lambda} f(X(0)) d \mathfrak{M}[X] .
$$

3. Мембранные амплитуды как точные квантовые волновые функции. В предыдущих формулах вся содержательная часть вычисления квантовой эволюции скрыта за значком интеграла по путям. Однако, в некоторых случаях от интеграла удается избавиться. Грубо говоря, это происходит, когда метод стационарной фазы дает точное значение интеграла, а не просто его асимптотику. Подобное, как известно, имеет место в формулах типа Дейстермаата-Хекмана или в формулах локализации. Следующая теорема расширяет класс таких примеров и задает виковский символ некоторых специальных квантовых операторов эволюции в чисто геометрических (т.е. классических) терминах.

Введем на $\mathfrak{X}$ меру $d m^{\prime} \stackrel{\text { def }}{=}\left(M^{2} / \operatorname{det} g_{1}\right) d \bar{z} d z$, где $M-$ это локальная плотность меры $d m=M d \bar{z} d z$. Обозначим через $\gamma^{t}$ поток гамильтонова векторного поля $\operatorname{ad}(H)$ на $\mathfrak{X}$, порожденного вещественной функцией $H$ и симплектической формой

$$
\omega_{0}=i g_{0} d \bar{z} \wedge d z \text {. }
$$


Следуя [6], [9], определим квантовую деформащию символа $H$ по формуле $H_{1} \stackrel{\text { def }}{=}$ $H-i \operatorname{div}^{\prime}\left(\operatorname{ad}(H)_{+}\right) / 2$. Здесь $\operatorname{ad}(H)_{+}-$это $(1,0)$-компонента векторного поля $\operatorname{ad}(H)$ относительно комплексной поляризации, а дивергенция $\mathrm{div}^{\prime}$ берется относительно меры $d m^{\prime}$. Положим

$$
\begin{gathered}
\Delta^{t}(x)=\left|\frac{\mathscr{D} z^{t}}{\mathscr{D} z}\right|\left(\frac{\operatorname{det} g_{1}\left(\gamma^{t}(x)\right)}{\operatorname{det} g_{1}(x)}\right)^{1 / 2} \\
\nu^{t}(x)=\frac{i}{4} \frac{\partial}{\partial t} \ln \frac{\mathscr{D} z^{t} / \mathscr{D} z}{\mathscr{D} \bar{z}^{t} / \mathscr{D} \bar{z}}-\frac{1}{2} \operatorname{Im}\left[\left(\frac{\partial}{\partial z} \ln \operatorname{det} g_{1}\right)\left(\gamma^{t}(x)\right) \dot{z}^{t}\right],
\end{gathered}
$$

где $z \equiv z(x), z^{t} \equiv z\left(\gamma^{t}(x)\right)$. Функцию $\Delta^{t}$ мы называем модулярной функцией, а

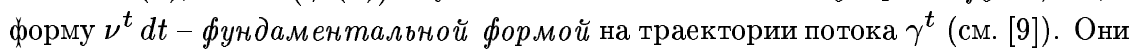
допускают инвариантное геометрическое описание [7], [10].

Теорема 3.1. Пусть задано квантование П комплексного многообразия $\mathfrak{X}$, и пусть квантовая метрика представима в виде (1.3). Предположим, что гамильтонов поток $\gamma^{t}$, порожденный функиией $H$ и симплектической формой (3.1), сохраняет поляризачию на $\mathfrak{X}$. Тогда

$$
\operatorname{tr}\left(e^{-i t \widehat{H}} \Pi(x)\right)=\exp \left\{i \int_{\Sigma^{t}(x)} \omega-i \int_{0}^{t} \nu^{\tau}(x) d \tau-i t H(x)\right\} \Delta^{t}(x)^{1 / 2} .
$$

Здесь $\Sigma^{t}(x)-$ это мембрана $\Sigma[X]$ с граничей (2.3), в которой $X=\left\{\gamma^{\tau}(x) \mid\right.$ $0 \leqslant \tau \leqslant t\}$ - это траектория потока $\gamma^{t}$.

В случае функций $H$, чьи гамильтоновы потоки не сохраняют поляризацию, в формуле типа (3.2) для ковариантного символа оператора эволюции присутствует еще дополнительная амплитуда, подчиненная некоторому уравнению. Это новое квантовое уравнение (инвариантно определенное на графике гамильтонова потока) элементарно решается в квазиклассическом приближении, см. [6], [9], [10].

Московский институт электроники и математики

Поступило E-mail: karasev@qds.amath.msk.ru

27.06 .96

\section{СПИСОК ЦИТИРОВАННОЙ ЛИТЕРАТУРЫ}

1. Klauder J. R. // J. Math. Phys. 1963. V. 4. P. 1058-1073. 2. Березин Ф. A. // Изв. АН СССР. Сер. матем. 1972. Т. 36. С. 1134-1167. 3. Simon В. // Comm. Math. Phys. 1980. V. 71. P. 247-276. 4. Переломов А. М. Обобщенные когерентные состояния и их применения. М.: Наука, 1987. 5. Rawnsley J. H. // Quart. J. Math. Oxford. 1977. V. 28. P. 403-415. 6. Karasev M. V. // Quantiz., Coherent States and Complex Struct. / ed. J.-P. Antoine et al. N. Y.: Plenum, 1995. P. 185-199. 7. Karasev M. V. // Russ. J. Math. Phys. 1993. V. 1. №4. P. 523-526. 8. Karasev M. V. // Russ. J. Math. Phys. 1994. V. 2. № 4. P. 445-462. 9. Karasev M. V. // Russ. J. Math. Phys. 1995. V. 3. №3. P. 393-400. 10. Karasev M. V. // Contemp. Math. 1994. V. 179. P. 83-113. 11. Klauder J. R. // Ann. Phys. 1960. V. 11. P. 123-168. 12. Klauder J. R. // Phys. Rev. Lett. 1986. V. 56. P. 897-899. 13. Cadavid A. C., Nakashima M. // Lett. Math. Phys. 1991. V. 23. P. 111-123. 